\title{
An Efficient Mobile Sink based Data Collection Model for Clustered based Wireless Sensor Network
}

\author{
Naveen Ghorpade $^{1}$, Dr. P. Vijaykarthik ${ }^{2}$ \\ ${ }^{1}$ Research Scholar, VTU, CSE Branch,Sir MVIT, Bangalore, Karnataka, India \\ ${ }^{2}$ Research Supervisor, VTU, ISE Department,Sir MVIT, Bangalore, Karnataka, India
}

\begin{abstract}
The Wireless Sensor Network (WSN) is considered to be a core component of tomorrow's real-time data communication networks, such as the Internet of Things (IoT). Modern networks need low-latency and high-throughputs in real-time due to a heterogeneous network. The availability of low-latency real-time data access incurs energy costs from the sensor systems. Clustering helped in maintaining the scalability and energy usage of sensors. However, it incurs overhead of the independent cluster head and sensor device within the close range of the sump pump. Since it would take longer transmission and recovery time. This Mine Research Paper introduces an Accessible Mobile Sensor Dependent Data Collection (EMSDC) Model for Cluster Based WSN (CWSN). Experiments are carried out to verify the efficiency of EMSDC and to equate it with the existing versions. The findings of the Latency and Overhead benchmarks demonstrated a lot of progress over the state-of-the-art versions.
\end{abstract}

Keywords - Bigdata, Clustering, Heterogeneous Wireless sensor network, IoT, Mobile sink.

\section{INTRODUCTION}

Advances in wireless networking, device, and micro-electronic technology have allowed miniaturisation of relatively low-cost, multifunctional sensors. These sensors are typically activated by shifting physical factors of the atmosphere such as temperature, humidity, and pressure. The calculated data is normally forwarded to the data collector using a cooperative approach and the collector may transmit the data to a remote server for further review. Besides, self-organizing systems may shape self-organizing sensor networks (SOSWSNs) [4]. The desirable characteristics of WSNs, such as fast deployment, strong fault tolerance, self-organization, real-time data transport, etc., render them ideal for deployment in unsanitary or aggressive conditions. WSN's are applied in commercial goods line control, agricultural, and wildlife surveillance [4], [5].

Sensors are primarily operated by batteries which are draining since they have to be recharged constantly. One of the better choices for energy scarcity in WSNs is to outfit the sensors with highcapacity batteries. However, the efficiency of this battery isn't going good and it can't be changed.
Therefore, the electricity crisis will only be dealt with by following energy-efficient protocols or strategies. Many experts have worked hard on the topic of energy management and energy balance, and has produced excellent results. Clustering technology significantly reduces the energy consumed by sensor nodes by splitting them into clusters according to certain special laws. We also addressed LEACH for $\mathrm{CCs}$ and further improvement is taking place because of LEACH. [7] addressed a fuzzy dependent algorithm to maximise energy efficiency of sensor network for broader network. However, one of the base stations in the cluster dies quite soon. As a consequence, the lifespan of the sensor are impacted. To address(8) presented Fuzzy Logic dependent clustering model. The scale of the sensor network was expanded with the inclusion of distributed sensors. The clustering model is constructed considering the homogenous networks. In this respect, routing strategies must consider the heterogeneity necessity of WSNs and their implementations (GAUS). These methods have been used for data collection [13], (forecasting) [14], clustering [17], [18], and evolutionary [16], [17] for growth. Both these algorithms have low energy loss but cause latency. 
In this way, Sink Mobile can be used in WSNs in a good manner. In mobile sink-supported WSNs, the sink is normally borne by intelligent vehicles across the sensing area and it travels freely across it. Here are few benefits of the revolutionary sink smartphone technology. The "hot spots" question may be considerably ameliorated with the rotation of the drain. Areas near the sink are typically high traffic areas, and when the sink shifts, these congestions often shift. Sensors would be spread uniformly to monitor electricity use. Next, a decrease in transmission distance will diminish the overall energy demand. Through usage of mobile sink, latency of the network can be minimised and throughput of the network can be improved. One special requirement of net access remains, including in the situation where sensors do not have connectivity. The incorporation of sink versatility creates several problems [14]. It is important to make it possible for network controllers to predict the position of the mobile sink. The moving pattern of the knowledge sink should be well built with the local nodes to collaborate for data transmission. A lot of smartphone sink dependent routing models have been established in recent years [22] [20] [21]. These techniques yield large maintenance overhead. Moreover, it's also accurate that a mobile sink dependent routing model operates. [23] proposed a routing algorithm for ad hoc wireless sensor networks. In this scenario, the mobile sink gathers data from $\mathrm{CH}$ unit. A slower clonal expansion into $\mathrm{CH}$ would create a strain in $\mathrm{CH}$. That may be a cause for bad company results. In this research paper we introduce the CWSN approach to capture data easily from mobile terminals. In Emergency Medical Services district committee first nominated candidates are chosen. Therefore, several RP nodes are chosen for each $\mathrm{CH}$. Then, this RP node is used as a rendezvous node for mobile sink to broadcast mobile information. This would contribute to improved network efficiency including the reduction of routing duration, contact overhead, and packet transmission delay.

The Contribution of research work is as follows:
- This paper proposed an effective mobile sink data collection model focused on clustered WSN.

- The EMSDC eliminates routing duration, overhead contact and packet transmission latency relative to the current WSN routing model.

The remainder of the document will be structured as follows. The proposed successful mobile sink data collection model for CWSN is discussed in Section II. The experimental analysis is carried out in the penultimate segment. Final and potential work is defined in the last section.

\section{EFFICIENT MObILE Sink BASED DATA} Collection Model For Clustered WSN

Mobile sink data collection software has been proposed in this portion of the work. Next, this work discusses the energy rule used. Secondly, make a choice on how to manage the route ahead by choosing the right node. Lastly, I want to discuss a way to obtain data traffic data.

The energy estimation process of the unit is as follows. Here it is measured the energy dissipation of sensing, contact and processor for characterising energy usage. The overall sum of energy dissipated by the three units is the total energy dissipated by each device. Thus the cumulated energy demand is calculated as follows.

$$
C_{\mathbb{E}}=K_{\mathbb{E}}+L_{\mathbb{E}}+D_{\mathbb{E}}
$$

This value indicates the sum of energy dissipation of all these processes. The sensor system to conserve energy turned off their sensing devices. Energy expended when transitioning from ON mode to OFF mode and vice versa. Another way to measure energy dissipation of sensing device is as follows.

$$
K_{\mathbb{E}}=K_{0 \rightarrow 1}+K_{1 \rightarrow 0}+K_{1 \rightarrow 1}
$$

where $K_{0 \rightarrow 1}$ is energy dissipation from $O F F$ to $O N$, $K_{1 \rightarrow 0}$ is energy dissipation from $O N$ to $O F F$ and $K_{1 \rightarrow 1}$ is energy dissipation for sensing. 
Similarly, the energy dissipation of processing unit $L_{\mathbb{E}}$ is computed as follows

$$
L_{\mathbb{E}}=L_{0}+L_{1}
$$

where $L_{0}$ is energy consumed in each mode, and $L_{1}$ is the energy necessary for transition from one mode to another. Therefore, the energy dissipation depends upon mode in which it is in, i.e. the processing unit is composed of following three modes, idle, sleep and running. The radio communication energy dissipation $D_{\mathbb{E}}$ can be computed considering that when $q$ bits is transmitted over distance $M$ is given as follows

$$
\begin{aligned}
& D_{\mathbb{E}}=D_{\overline{\mathcal{T}}}(q, M) \\
& = \begin{cases}q K_{\mathcal{E}}+q \alpha f(M)^{2} & M<M_{0} \\
q K_{\mathcal{E}}+q \alpha x(M)^{4} & M>M_{0}\end{cases}
\end{aligned}
$$

where $D_{\mathbb{E}}$ is the energy dissipation by transceiver circuit, $\alpha x$ and $\alpha f$ is the amplification feature for multipath and free space propagation, $M_{0}=\sqrt{\frac{q \alpha f}{q \alpha x}}$ is the distance factor. $D_{\mathcal{T}}$ and $D_{\mathcal{R}}$ is the energy dissipation for transmitting and receiving $q$ amount of bits over distance $M$. Our energy model offers more ideal energy transfer in heterogeneous wireless sensor networks.

Cluster head selection: First the sensor device are placed across network and position of its is known in prior. Then, the cluster head selection process is initialized. The $\mathrm{CH}$ is selected using multi-objective thresholding parameter (TP) $V_{\mathbb{N C}}^{n}$. The multi objective parameter for $\mathrm{CH}$ selection are coverage time $\delta J_{\mathbb{N} \mathbb{C}}^{\mathbb{C}}(u, u+t)$, connection time $\delta E_{\mathbb{N} \mathbb{C}}^{\mathbb{C}}(u, u+$ $t)$, RSS and connectivity $H_{\mathbb{N C}}^{n}$. The TP for selecting $\mathrm{CH}$ are obtained considering the SD with maximum CG, residual energy, better connection time, and RSS which can described using below equation

$$
\begin{aligned}
V_{\mathbb{N} C}^{n}=G_{C}+ & \left(\frac{O_{\mathbb{C}}^{c}}{O_{\mathbb{N}}^{\mathbb{M}}-O_{\mathbb{C}}^{c}} * \frac{F_{\mathbb{N}}^{\mathbb{M}}-F_{\mathbb{C}}^{c}}{F_{\mathbb{N}}^{\mathbb{M}}}\right. \\
& \left.* \frac{W_{\mathbb{N}}^{\mathbb{M}}-W_{\mathbb{C}}^{c}}{W_{\mathbb{N}}^{\mathbb{M}}+W_{\mathbb{C}}^{c}} * \frac{S_{\mathbb{N}}^{\mathbb{M}}-S_{\mathbb{C}}^{c}}{S_{\mathbb{N}}^{\mathbb{M}}+S_{\mathbb{C}}^{c}}\right) .
\end{aligned}
$$

Using above equation the sensor device with maximum $V_{\mathbb{N C}}^{n}$ (i.e., TP) are selected as $\mathrm{CH}$. In Eq. (5) a Boolean parameter $G_{C}$ is introduced by adding it with multiplicative factor of multi-objective parameter such as residual energy, adjacent SD size, speediness of SD, coverage distance. When $G_{C}=1$ then the sensor device was the $\mathrm{CH}$ of previous round. Further, if $G_{C}=0$ then the $\mathrm{SD}$ is having higher chance of getting selected as cluster head for current round in accordance with TP. $O_{\mathbb{C}}^{c}$ depict the $\mathrm{CG}$ size, $O_{\mathbb{N}}^{\mathbb{M}}$ depicts the maximal $\mathrm{CG}$ for each round, $F_{\mathbb{N}}^{\mathbb{M}}$ depicts the SD preliminary energy level, $O_{\mathbb{N}}^{\mathbb{M}}$ depicts the maximal amount of $\mathrm{CG}$ for each round, $W_{\mathbb{N}}^{\mathbb{M}}$ depicts maximal speediness of $\mathrm{SD}, W_{\mathbb{C}}^{c}$ describes the current speediness of $\mathrm{SD}, S_{\mathbb{N}}^{\mathbb{M}}$ depicts the maximal coverage radius $(\mathrm{CR})$ of $\mathrm{SD}, S_{\mathbb{C}}^{c}$ describes the current $\mathrm{CR}$ of SD. The proposed TP aid in selecting $\mathrm{CH}$ with less mobile nature, with maximal coverage distance (CD) and with high number of CG sensor device.

Rendezvous node selection and path construction: The mobile sink route was built with RPN. The task of resource planner is to collect the aggregated data from cluster heads and send to MS. The resource planner is chosen by BS. This is analogous to the threshold filtering process. the RPN are picked. An development was made to achieve improved performance of packet transmission and energy consumption. (1) The multi-objective parameter considered are packet length, buffer capacity, link time, and coverage time. Routing decision for $\mathrm{CH}$ has to be selected within one-hop neighbour of RPN. This is to reduce network overhead of $\mathrm{CH}$ and increase capacity performance of WSN. Finally, by utilising RPN the MSP is retrieved. The introduction of RPN can be implemented if maintenance service level has been done considering present coverage and versatility of electric cars using following equation.

$$
\begin{aligned}
U_{\gamma \mu}^{q}=I_{\gamma}+\left(\frac{F_{O}^{\mathbb{M}}-F_{\mu}^{c}}{F_{O}^{\mathbb{M}}} * \frac{W_{O}^{\mathbb{M}}-W_{\mu}^{c}}{V_{O}^{\mathbb{M}}+W_{\mu}^{c}}\right. \\
\\
\left.* \frac{S_{O}^{\mathbb{M I}}-S_{\mu}^{c}}{S_{O}^{\mathbb{M}}+S_{\mu}^{c}}\right)
\end{aligned}
$$

where $I_{\gamma}$ depicts the hop size of $\gamma$ with respect to $\mathrm{BS}$ in MSP, $\gamma$ are utilized to establish adjacent device 
in MSP, $F_{O}^{\mathrm{MI}}$ depicts the preliminary energy level of $\mathrm{SD}, F_{\mu}^{c}$ depicts the present energy level of $\mu$, the parameter $\mu$ are utilized it establish the RPN considering adjacent or next hop SD, $W_{O}^{\mathrm{MI}}$ depicts the maximal speediness of $\mathrm{SD}, W_{\mu}^{c}$ depicts current speediness of $\mu, S_{O}^{\mathbb{M}}$ depicts maximal CR of SD, $S_{\mu}^{c}$ depicts the current CR of SD. The usage of RPN aid in minimizing overhead of communication (i.e., packet transmission) and energy overhead (i.e., energy incurred for hop transmission) of WSN. For enhancing the network lifetime (NL) of WSN every round a new RPN and $\mathrm{CH}$ is chosen. Further, it should be noted RPN does not take part in sensing operation. As a result, the RPN does not belong to any of the cluster and does affect communication of any CG. Post establishing MSP the data are collected by mobile sink from each RPN node.

The objective of our routing design is to find the low latency path with minimal energy consumption. In each cluster, the sensor device at longer distance from base station or cluster head use path/link selection parameter $L_{\mathcal{M}}$ to find the best path to cluster head or base station which is as follows

$$
L_{\mathcal{M}}=\mathcal{E}_{v}+\mathcal{G}_{l}+\overline{\mathcal{G}}_{l}+\mathbb{L}_{l}
$$

where $\mathcal{E}$ is the residual energy of any energy category, $v$ is the sensor node at a different category, $\mathcal{G}_{l}$ is the expected hop number, $\overline{\mathcal{G}_{l}}$ is the inverse of expected hop number and $\mathbb{L}_{l}$ is the minimum data loss parameter.

where $\mathcal{G}_{l}$ is obtained using following equation

$$
\mathcal{G}_{l}=\sum_{t \in l} \frac{1}{\mathcal{S D}(t) * \mathcal{D} \mathcal{S}(t)},
$$

where $\overline{\mathcal{G}}_{l}$ is obtained using following equation

$$
\overline{\mathcal{G}}_{l}=\sum_{t \in l} \mathcal{S D}(t) * \mathcal{D} \mathcal{S}(t),
$$

where $\mathbb{L}_{l}$ is obtained using following equation

$$
\mathbb{L}_{l}=\prod_{t \in l} \mathcal{S} \mathcal{D}(t) * \mathcal{D} \mathcal{S}(t)
$$

where $\mathcal{S} \mathcal{D}(t) * \mathcal{D} \mathcal{S}(t)$ is the data delivery ratio on link $t$ of transmission path $l$ from destination to source $\mathcal{D} \mathcal{S}(t)$ and from source to destination $\mathcal{S}(t)$.

The beacon sends the beacon request message to neighbouring beacons and respond with beacon reply message to the beacon request message. So we know how to get from P or System A to Device B. Multipath connectivity assistance can be used to reduce network congestion, enhance network stability, maintain resilience and raise fault tolerance and also boost load balancing, enhance network energy usage and prolong network lifespan. Once the routes are found, the right route is picked. Since the proposed EMSDC model does not only conserve electricity, but also minimises latency. Besides, minimise network interference and balance energy in the network.

proposed EMSDC and LEACH is introduced using Windows programming language using Visual Studio 2008 and above. The parameter settings of the virtual model are described in Table I.
TABLE I. These are the structures that the researcher aims to study. This is an example of remote sensing by the SENSORIA simulator. The algorithm of

TABLE II. SIMULATION PARAMETER CONSIDERED

\begin{tabular}{|c|c|}
\hline Network Parameter & Value \\
\hline Network size & $100 \mathrm{~m} \times 100 \mathrm{~m}$ \\
\hline Number of base station/Sink & 1 \\
\hline Number of sensor devices & $1000,2000,4000$ \\
\hline Radio propagation/Transmission range of each sensor device & $10 \mathrm{~m}$ \\
\hline Sensing range of each device & $6 \mathrm{~m}$ \\
\hline Initial energy of each sensor devices & $0.1 \mathrm{Joules}(\mathrm{j})$ \\
\hline Radio energy dissipation & $50 \mathrm{nj} / \mathrm{bit}$ \\
\hline
\end{tabular}




\begin{tabular}{|c|c|}
\hline Control packets (CP) size & $248 \mathrm{bits}$ \\
\hline Data packets (DT) size & $2000 \mathrm{bits}$ \\
\hline Data transmission speed & $100 \mathrm{bit} / \mathrm{s}$ \\
\hline Bandwidth (BW) & $10000 \mathrm{bit} / \mathrm{s}$ \\
\hline Idle energy consumption (Eelec) & $50 \mathrm{nj} / \mathrm{bit}$ \\
\hline Amplification energy (Emp) & $100 \mathrm{pJ} / \mathrm{bit} / \mathrm{m} 2$ \\
\hline
\end{tabular}

a) Network Lifetime Efficiency Review $85.27 \%$ was obtained over LEACH considers the considering Complete System Death:

Experiments are performed on more effective than double redundancy schemes. Perform a network lifetime assessment on the basis of network application region. 2. A lifetime efficiency gain of $68.36 \%, 76.64 \%, 83.16 \%, 84.11 \%, 84.05 \%$ and usage of 500, 600, 700, 800, 900 and 1000 sensors. A history accomplishment is $70-80 \%$ gain over LEACH. It is obvious from Fig. As the scale of the sensor system is greater, the lifetime output reduces, but the EMSDC is constant independent of sensor device size.

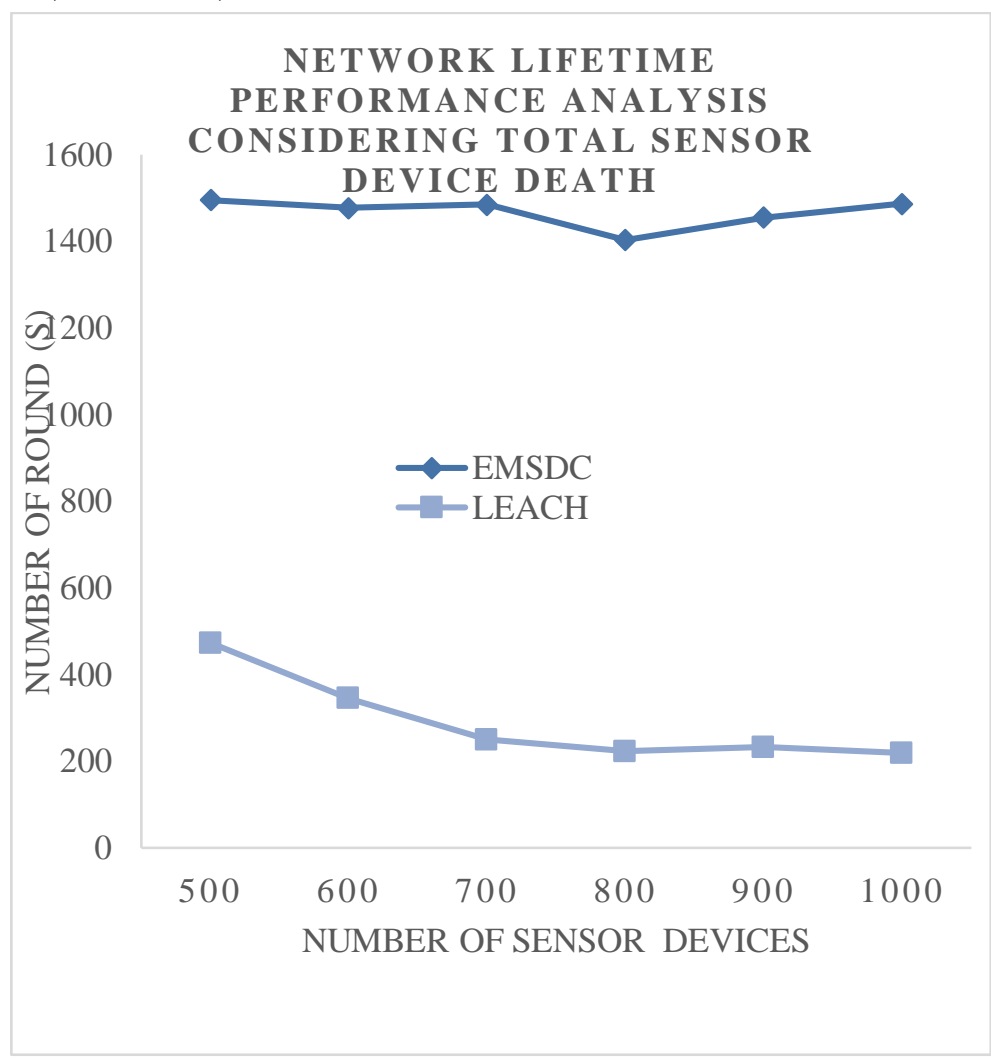

Fig. 2. Network lifetime performance analysis considering varied sensor devices

b) Communication Overhead Performance Analysis:

Experiments are performed to determine of the contact network of LSDC's EMS over LEACH. The efficiency is tested by varying network measurement sensor parameters. 4. A contact overhead of $16.81 \%$, $9.01 \%, 39.78 \%, 34.39 \%, 40.57 \%$ and $51.54 \%$ is accomplished by LEACH over EMSDC considering 500, 600, 700, 800, 900 and 1000 sensor devices respectively. An average reduction of 32.02 percent is accomplished over LEACH. It is obvious from Fig. EMSDC and LEACH connectivity overhead dramatically improves as computers are rendered bigger. 


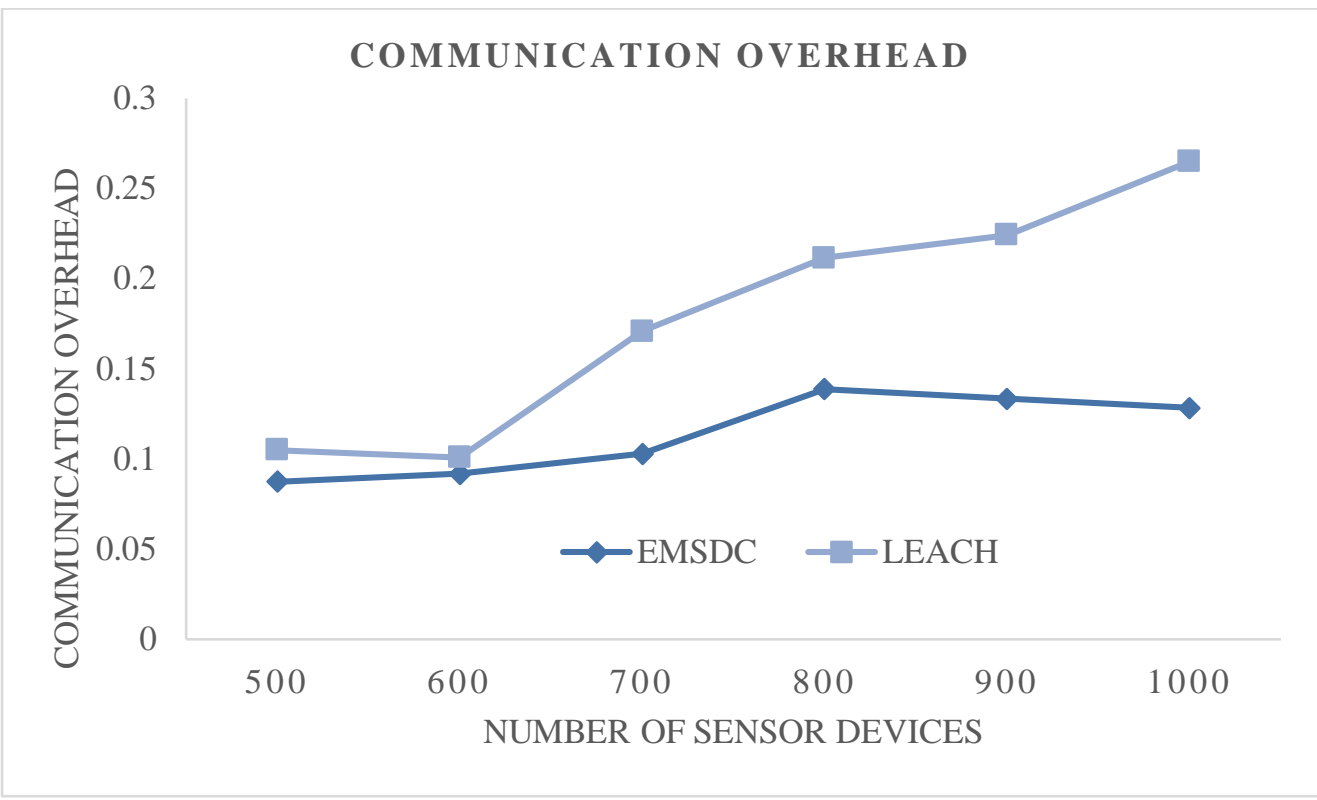

Fig. 3. Communication overhead efficiency review considering a range of sensor instruments

c) Routing Overhead Performance Analysis:

Experiments have been undertaken to assess the efficiency of the proposed network in LEACH-based EMSDC. Network subnetwork hop selection overhead assessment is carried out by modifying the network sensor system according to fixed network location. 4. A decrease of $56.48 \%, 51.45 \%, 49.69 \%$, $57.69 \%, 51.19 \%$ and $41.94 \%$ is accomplished by EMDC over LEACH. The total routing overhead reduction accomplished by Sendai Airport Company in December has been 51.41 percent. It is obvious from Fig. The size of the sensor devices raise does not contribute to increased EMSDC and LEACH routing overhead.

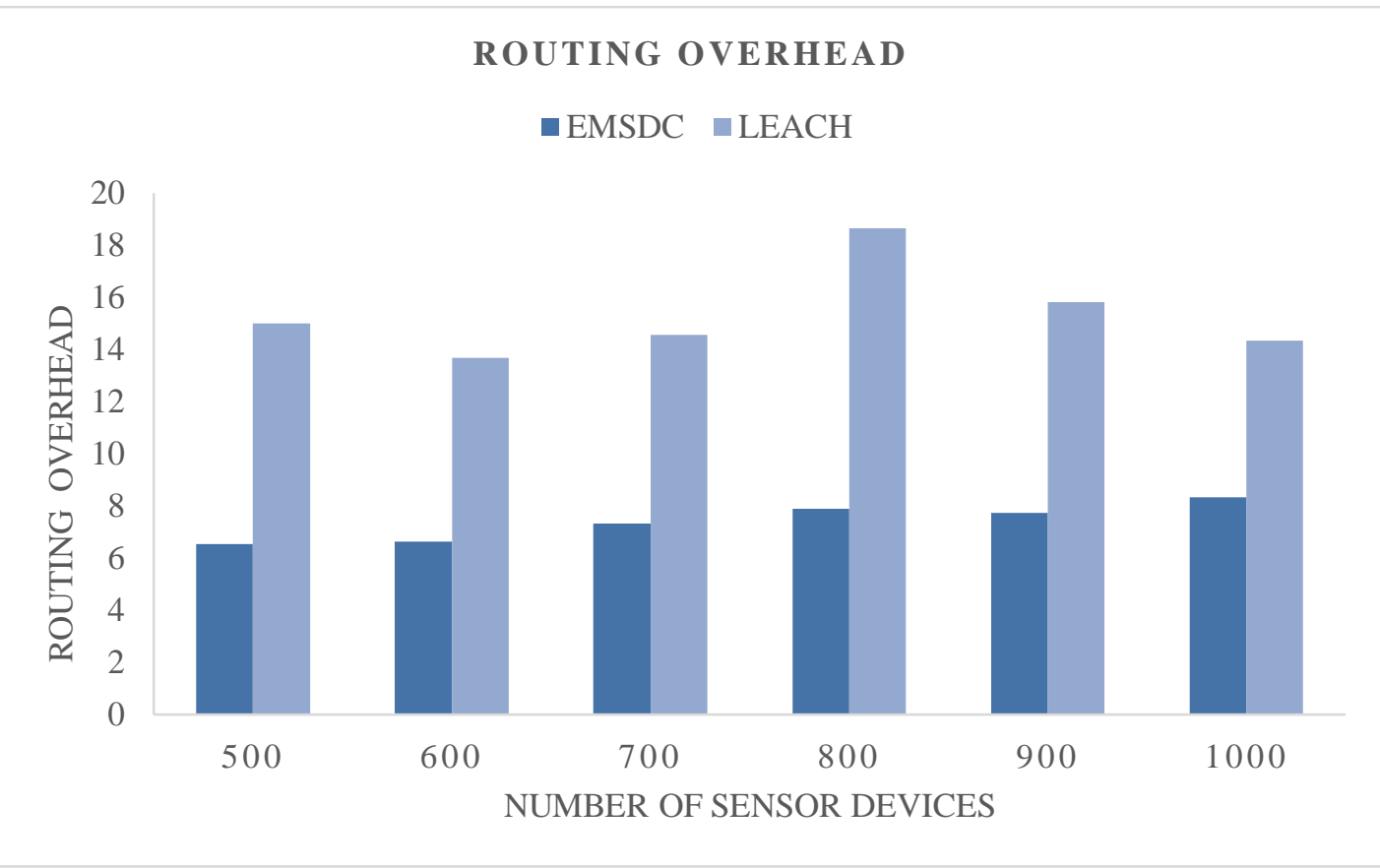

Fig. 4. Routing overhead performance analysis considering varied sensor devices.

d) Data Processing Latency Performance Analysis:
Experiments are performed to test the latency overhead efficiency of proposed LEACH over EMSDC. The network data analysis latency 
efficiency is checked by changing the sensor position in terms of geographic zone. 6. EMSDC finds that a latency reduction of $57.76 \%, 52.34 \%, 51.05 \%$, $59.46 \%, 52.28 \%$ and $51.54 \%$ with regard to degree of error rate of 500, 600, 700, 800, 900 and 1000 sensor devices respectively, is achieved. The total increase in overall machine latency by EMSDC by 53.22 percent relative to $\mathrm{LEACH}$. It is obvious from Fig. When the size of an EMSDC and Leach sensor system is raised, the processing delay often increases.

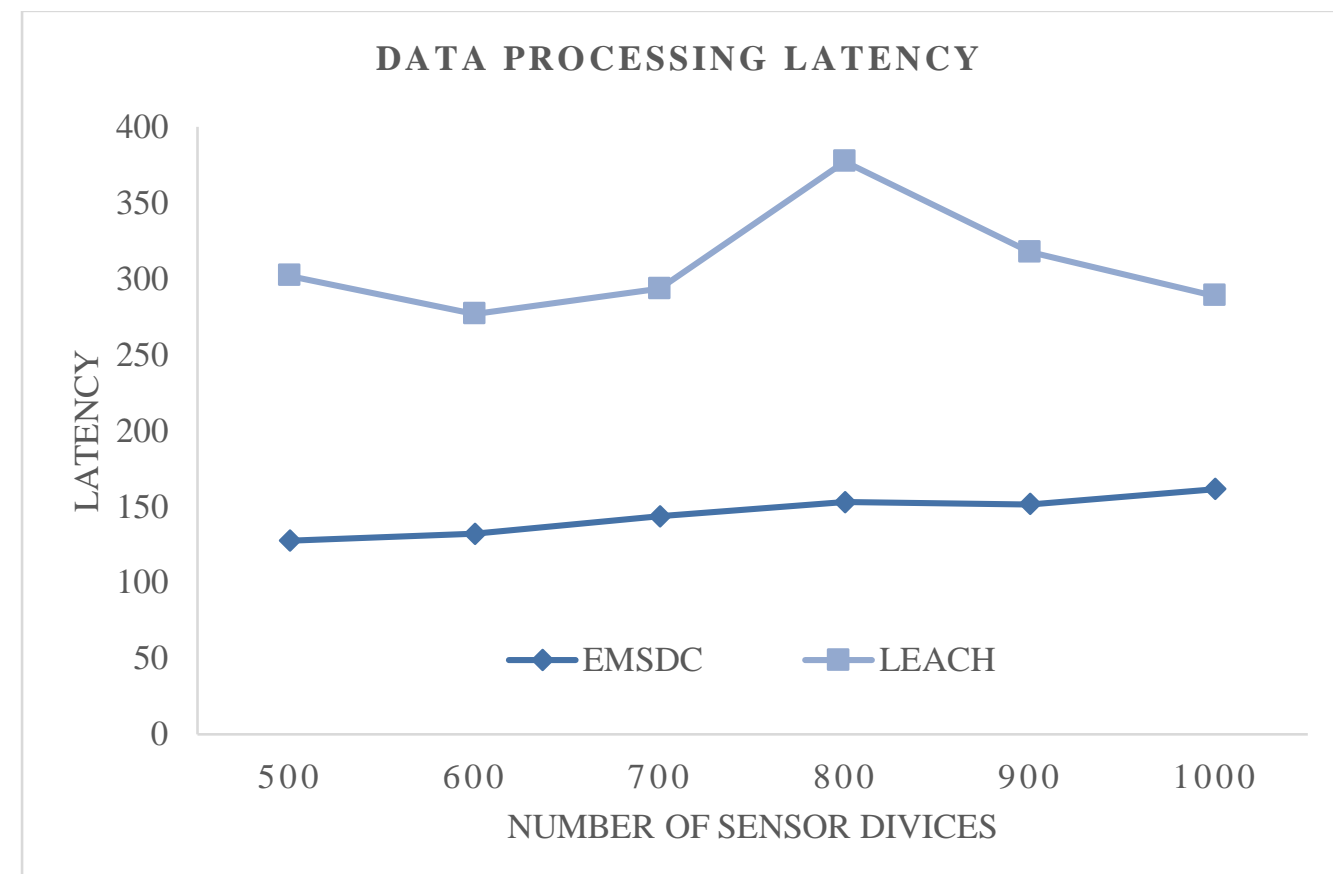

Fig. 5. Data processing latency performance analysis considering varied sensor devices

The overall result indicates that the EMSDC model has accomplished substantial change in the efficiency of the network over the state-of-the-art models[7],[8],[16],[18] and[20] considering the first node death and final node death.

\section{CONCLUSION}

Minimizing the resources consumed by one's sensor system is more beneficial. Numerous methods have been advanced to reduce energy usage in modern years. IoT will need data access within low latency time. The current ones are not perfect for handling it. These implementations are chosen considering heterogeneous wireless sensor network. Very little investigation on heterogeneity has been carried out so far. With EMSDC, energy usage is minimised, and the lifespan and latencies are maximised. The network lifetime is improving of 80.26 percent (Average increase in network lifetime over LEACH) by EMSDC over LEACH over varied sensors is appraised. EMSDC decreases communications delay and routing overhead by $51.41 \%$ and improves message processing performance by $32.02 \%$ over LEACH protocol. Overall, the findings achieved indicate the utility of EMSDC over the state-of-art models [7], [8], [16], [18], and [20]. Future study will analyse performance by using EMSDC; will propose offering protections for EMSDC.

\section{REFERENCES}

[1] M. R. Palattella, M. Dohler, A. Grieco, G. Rizzo, J. Torsner, T. Engel, and L. Ladid, "Internet of things in the 5G era: Enablers, architecture, and business models," IEEE Journal on Selected Areas in Communications, vol. 34, pp. 510-527, Mar. 2016.

[2] M. Simsek, A. Aijaz, M. Dohler, and J. Sachs, "5G-enabled tactile internet," IEEE Journal on 
Selected Areas in Communications, vol. 34, pp. 460-473, Mar. 2016.

[3] S. Cirani and M. Picone, "Wearable computing for the internet of things," It Professional, vol. 17, pp. 35-41, Sep. 2015.

[4] A. Zanella, N. Bui, A. Castellani, and L. Vangelista, "Internet of things for smart cities," Internet of Things Journal IEEE, vol. 1, pp. 2232, Feb. 2014.

[5] W. B. Heinzelman, A. P. Chandrakasan, and H. Balakrishnan, "An application-specific protocol architecture for wireless microsensor networks," IEEE Trans. Wireless Commun., vol. 1, no. 4, pp. 660-670, Oct. 2002.

[6] D. V. Puspalata and P. Nayak, "A clustering algorithm for WSN to optimize the network lifetime using type-2 fuzzy logic model," in Proc. 3rd Int. Conf. Artif. Intell., Modeling Simulations (AIMS), Kota Kinabalu, Malaysia, Dec. 2015, pp. 53-58.

[7] P. Nayak and A. Devulapalli, "A Fuzzy LogicBased Clustering Algorithm for WSN to Extend the Network Lifetime," in IEEE Sensors Journal, vol. 16, no. 1, pp. 137-144, Jan.1, 2016.

[8] P. Nayak and B. Vathasavai, "Energy Efficient Clustering Algorithm for Multi-Hop Wireless Sensor Network Using Type-2 Fuzzy Logic," in IEEE Sensors Journal, vol. 17, no. 14, pp. 44924499, July15, 152017.

[9] U. Srinivasan and B. Arunasalam, "Leveraging big data analytics to reduce healthcare costs," IT Prof., vol. 15, no. 6, pp. 21-28, Nov./Dec. 2013.

[10] N. Sun, J. G. Morris, J. Xu, X. Zhu, and M. Xie, "iCARE: A framework for big data-based banking customer analytics," IBM J. Res. Develop., vol. 58, nos. 5/6, pp. 4:1-4:9, 2014.

[11] C. Sun, R. Gao, and H. Xi, "Big data based retail recommender system of non E-commerce," in Proc. Int. Conf. Comput., Commun. Netw. Technol., pp. 1-7, 2014.
[12] [Online]. Available: http://www.ibm.com/bigdata/au/en/big-data-andanalytics/ operationsmanagement.html

[13] K. L. M. Ang; J. K. P. Seng; A. M. Zungeru, "Optimizing Energy Consumption for Big Data Collection in Large-Scale Wireless Sensor Networks With Mobile Collectors," in IEEE Systems Journal , vol.PP, no.99, pp.1-11, 2017.

[14] S. Rani, S. H. Ahmed, R. Talwar and J. Malhotra, "Can Sensors Collect Big Data? An EnergyEfficient Big Data Gathering Algorithm for a WSN," in IEEE Transactions on Industrial Informatics, vol. 13, no. 4, pp. 1961-1968, Aug. 2017.

[15] X. Liu, J. Li, Z. Dong and F. Xiong, "Joint Design of Energy-Efficient Clustering and Data Recovery for Wireless Sensor Networks," in IEEE Access, vol. 5, no. , pp. 3646-3656, 2017. 